\title{
Progress in the Nuclear Imaging Techniques for Cognitive Studies
}

\author{
Thirumurugan Elango ${ }^{1}$, Ramesh Naidu Annavarapu ${ }^{2}$ \\ Department of Physics, Pondicherry University, Puducherry-605014, India
}

\begin{abstract}
The recent advancement in the nuclear physics lead to a state of art nuclear equipment's where different kinds of isotopes like ${ }^{99 m} T c,{ }^{111} I n,{ }^{123} I,{ }^{133} \mathrm{Xe}$ are used as radioactive tracer in imaging to diagnose the diseases, Electric power generation, National security etc., This recent development paved the way for the nuclear physicist to go on to find different isotopes and methods to design different types of equipment which are used for various purpose. This Paper gives a gist of few recent developments and it's applications with an importance to Medical diagnosis with heavy ion beams or protons, Positron Emission Tomography (PET) and Magnetic resonance imaging (MRI).
\end{abstract}

Keywords - PET, MRI, Scintillators, Tracers, PET/MR

\section{Introduction}

The uses of Nuclear physics is growing in an exponential way. The Major areas are electricity generation, nuclear stockpile for national security, Radioactive dating, interplanetary spacecraft, smoke detectors in households, medical diagnosis with Positron emission Tomography (PET), Time -of-flight PET, Magnetic Resonance Imaging (MRI), and proton or heavy ion beam for cancer diagnosis. Recent progress in the nuclear imaging techniques for the spatial and temporal imaging of the whole body with the state of art nuclear imaging equipment using the radioactive tracers lead to a big leap in medical diagnosis of whole body imaging including the improvement in cognitive studies which would not be possible without Current advancement in Nuclear Physics. This paper mainly discusses the current progress in nuclear physics related to the medical diagnosis which is useful for cognitive studies.

\section{Technological Evolution}

The evolution of the nuclear physics started in 1789 by the discovery of the Uranium by Martin Kalproth but as a discipline separate from atomic physics was stated in 1896 by Henri Becquerel. As the world of nuclear physics was witnessing a tremendous advancement in Harnessing nuclear energy for electricity and atomic bomb using uranium (U-235) and plutonium. At the same time, MRI and PET have also undergone tremendous advancements. A small timeline can give a gist of the evolution

The Magnetic Resonance Imaging (MRI) is due to the advancement of the NMR for analyzing the tissue. In other words, the use of advanced NMR for medical diagnosis is called MRI.

Table 1

\begin{tabular}{|l|l|}
\hline 1969 & The whole body MR Scanner Has been proposed by Damadian \\
\hline 1970 & The key discovery of T1/T2 difference in Cancer and Normal Cell by Damadian made MR studies worthy. \\
\hline Mar 1971 & T1/T2 difference overcome the X-Ray inability to view the vital organs \\
\hline Spr 1971 & Damadian outlines Voxel scanning method \\
\hline Sept 1971 & Gradient method Proposed \\
\hline Mar 1972 & The first patent filed for 3-D voxel by Voxel scanning \\
\hline Oct 1972 & 2-D scan achieved \\
\hline Mar 1973 & 2-D paper Published \\
\hline 1974 & 3-D scan Method proposed \\
\hline 1975 & Phase coding Proposed \\
\hline 1977 & First human Scan Achieved \\
\hline 1980 & $\begin{array}{l}\text { Phase coding applied using Spin wrap method, Damadian and FONAR introduced first Commercial MRI using } \\
\text { VOXEL method }\end{array}$ \\
\hline 1997 & Patent Upheld \\
\hline
\end{tabular}

PET is based on the principle that the opposing $511-\mathrm{KeV}$ photons originating because of the annihilation of emmitted positron with a nearby electron [7]. The table 2 shows a timeline of PET development. 


\section{Table 2}

\begin{tabular}{|l|l|}
\hline 1950 & $\begin{array}{l}\text { David E. Kuhl introduced the concept of transmission and emission tomography, } \\
\text { first demonstration of annihilation radiation for medical imaging by Gordon Brownell, Charles } \\
\text { Burnham[1] }\end{array}$ \\
\hline 1961 & First single plane PET scan by James Robertson and associates[2] \\
\hline $1968-1969$ & First instrument with logical extension of positron instrumentation in 2-D is completed \\
\hline 1970 & $\begin{array}{l}\text { First application of PC-I in tomographic mode was reported by distinguishing it from computed } \\
\text { tomographic mode[3] }\end{array}$ \\
\hline 1976 & 2-fluorodeoxy-D-glucose (2FDG) was administered in human volunteer \\
\hline 1980 & Concept of Time-Of-Flight PET is introduced \\
\hline 1990 & Bismuth germanate was used as major scintillator in PET \\
\hline $2000 \mathrm{~s}$ & An important component called lutetium oxyorthosilicate (LSO) has been developed[4] \\
\hline 2006 & First commercial TOF-PET Scanner is introduced[4,5,6] \\
\hline
\end{tabular}

\section{Basics and Usefulness of PET}

PET is known for its molecular imaging of biological functions. PET measures two annihilation protons which are obtained back to back from radioactive tagged tracer molecule. So the detection of both annihilated photon provides a high sensitive image with respect to PET counterpart imagers. In PET less amount of tracer molecules are injected due to its high sensitivity. So the Positron emission isotopes like C-11, O-15, , $\mathrm{N}-13, \mathrm{~F}-18$ are used in the Positron emission tomography. As well as these radioactive tagged tracers are shortlived and are less injurious to the person who are to be injected. Many of these isotopes can also be incorporated into the biological substrates like $\mathrm{C}_{6} \mathrm{H}_{12} \mathrm{O}_{6}$, Ammonia, $\mathrm{CO}_{2}$ etc., Though PET scan appears to have lot of noise and blurriness compared to that of the other imagers which can go up to $1 \mathrm{~mm}$ resolution. The PET are useful in Focal tracer concentration i.e. the accurate quantitative functional imaging due to its high sensitivity [7]

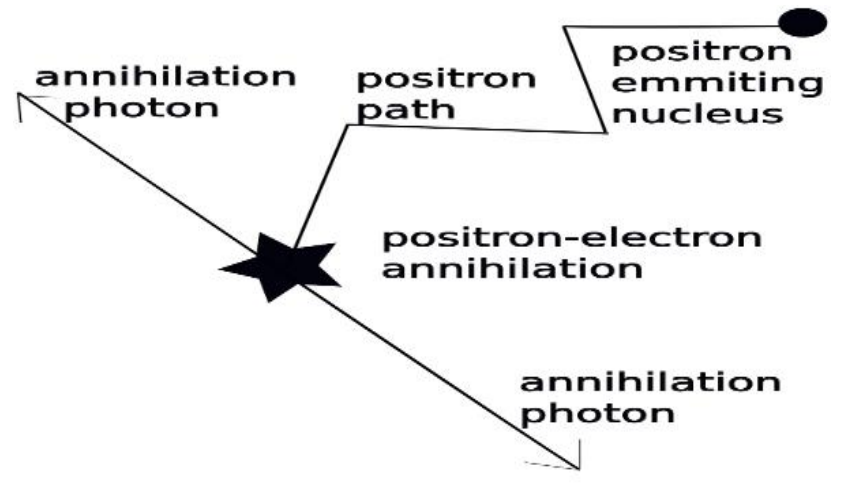

Figure 1

Fig 1 explains the concept of PET, to explain briefly if a radioactive iodine is injected into patients body it reaches the thyroid gland and it is absorbed in the area where more metabolic activities are seen. As they decay they give out positron, while the positron colliding with the electron the annihilation occurs which later gives out two beams of gamma rays. There are rings of radioactive detectors which takes up gamma rays which combines with the crystal detectors to give out the beam of a pulse of light. Which is then amplified by photomultiplier and the information is processed and analyzed in the computer. These are the basic working principle of PET. For PET imaging of the brain Fluorodeoxyglucose(FDG) is used.[7]

\section{Basic and Usefulness of MRI}

The simultaneous question when the topic MRI rises is how it is related to Nuclear Physics. The MRI's are advanced NMR which can detect the proton (hydrogen) nuclear spin which is partially polarized by subjecting it to a large magnetic field and then exiting the spins properly with Radio frequency radiation to detect back the weak signals which are emitted back from the magnetic interaction with the tissue or some biological objects.[8]

The MRI is able to see Hydrogen nuclei which became a boon for this field to advance in clinical diagnosis as $70 \%$ of the Human body is of water. Water $\left(\mathrm{H}_{2} \mathrm{O}\right)$ consists of two hydrogen atoms and one $\mathrm{O}$ atom. The MRI cannot record the oxygen atom and electrons of the hydrogen atom. So now MRI is pinned down to the nucleus of the Hydrogen atom. Here comes the complex concept of understanding the nucleus of the hydrogen which later used for imaging the various biological objects. The MRI obey the rules of Quantum physics as they are dealing with very very small particles. The concept of MRI goes along with a quantum concept i.e. the well-known 'spin'. Which goes according to the orientation of the nucleus. The hydrogen nuclei

\footnotetext{
National Conference on Current Advancements in Physics $3^{\text {rd }} \& 4^{\text {th }}$ February 2017 $62 \mid$ Page Department of Physics, St. John's College, Palayamkottai-627 002, Tamilnadu, India. DOI 10.9790/4861-17002036168
} 
in MRI is detected by resonance effect created by the strong electromagnets which give out the heavy and continuous magnetic field. This magnetic field aligns the spin of the hydrogen nuclei in its direction but the nuclei which have high energy are aligned in opposite direction. There are always more low energy nuclei than the high energy ones. This helps in concept of developing the MRI. When Radio Frequency coil disturbs the magnetic field the spin of low energy nuclei changes and goes to exited state becoming high energy nuclei. After RF signal is stopped the low energy nuclei which are turned into high energy nuclei returns back to low energy by releasing some energy. The released energy is converted into electrical signals in MRI with respect to time and later they are reproduced into images. The MRI cannot scan every part at a particular time. So the imaging is done in Slices with respect to time (the below blue-yellow-green arrows represents it).

In below Fig 2 Green coils are strong electromagnets, green lines are magnetic fields, blue, pink coils are RF coils, and red dots are hydrogen nuclei.

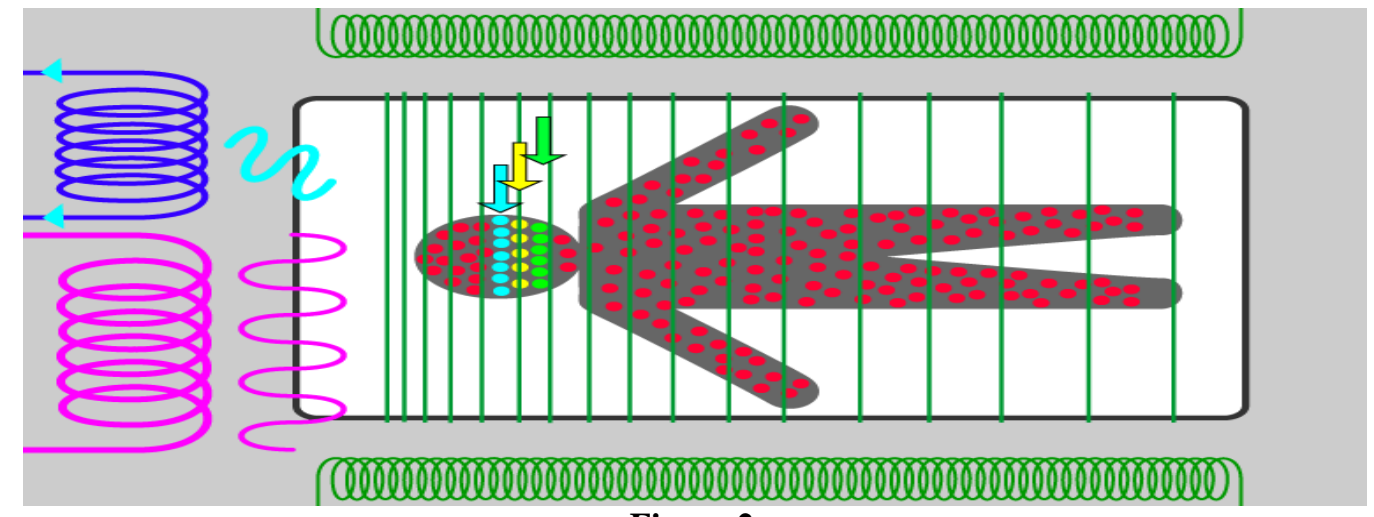

Figure 2

\section{Advancement In PET}

As per 2011 census, PET/CT application is 94\% in oncology, 3\% in cardiology, 3\% in neurology with in and around 1.85 million PET scan per year.[9,10]

\subsection{Advantage of PET over CT}

- reduction in the level of geometrical misses

- standardization of volume delineation

- minimization of radiation dose as new and efficient tracers are developed for PET[10,11]

The progress of PET cannot be fully explained without the scintillators. Scintillator detectors are the detector elements which are used to completely absorb the energy from the photon of $511 \mathrm{KeV}$ and convert it into tens of thousands of near visible or ultraviolet rays with nearly $1 \mathrm{eV}$ energy each.[7]

No of scintillation photon produced is directly proportional to the energy deposited by annihilation photon.

\subsection{There are many scintillators used in PET scanners few of them are}

- Thallium-doped sodium iodide $\mathrm{NaI}(\mathrm{Ti})$

- Bismuth germinate (BGO)

- Lutetium yttrium orthosilicate (LYSO)

- Gadolinium orthosilicate (GSO) [7]

\subsection{Few characteristics of scintillators are}

- light output which determines energy and spatial resolution

- Effective density determines scanner sensitivity

- Light decay time which determines scanner deadtime and random coincidence rate as well as ability to use in TOF-PET imaging.

\subsection{Important properties for PET photon detection are [7]}

- stopping power

- decay constant

- energy resolution

The Ideal detector of PET

National Conference on Current Advancements in Physics $3^{\text {rd }} \& 4^{\text {th }}$ February 2017 
- Should have high stopping power i.e. the crystals should completely absorb 511KeV Photon energy

- Should have the ability to determine the interaction location of the gamma ray in the detector to a Voxel should be high.

- should not reject the scattered events i.e. it should have good energy resolution

- should have very high timing resolution

The scintillators play a vital role in PET. Various scintillators are good in various important properties while lacking few of it. Properties [7,12]

Table 3

\begin{tabular}{|l|l|}
\hline Cost of material & $\mathrm{NaI}(\mathrm{Ti})<\mathrm{BGO}<\mathrm{LYSO}<\mathrm{GSO}$ \\
\hline Effective density & $\mathrm{NaI}(\mathrm{Ti})(3.67 \mathrm{~g} / \mathrm{cm} 3)<\mathrm{GSO}(6.71 \mathrm{~g} / \mathrm{cm} 3) \leq \mathrm{LYSO}(5.37 \mathrm{~g} / \mathrm{cm} 3)<\mathrm{BGO}(7.13 \mathrm{~g} / \mathrm{cm} 3)$ \\
\hline Light output & $\mathrm{BGO}(8 \%)<\mathrm{GSO}(16 \%)<\mathrm{LYSO}(75 \%)<\mathrm{NaI}(\mathrm{Ti})(100 \%)^{I}$ \\
\hline
\end{tabular}

1- with respect to $\mathrm{NaI}(\mathrm{Ti})$

NaI(Ti)- Thallium-doped sodium iodide

$\mathrm{GSO}-\mathrm{Gd}_{2} \mathrm{SiO}_{5}$ cerium doped Gadolinium oxy-orthosillicate

$\mathrm{BGO}-\mathrm{BI}_{4} \mathrm{Ge}_{3} \mathrm{O}_{12}$ Bismuth germanate oxide

LYSO - lutetium yttrium orthosilicate

The detector should be of high atomic number and high density is necessary for photoelectric conversion of the annihilated photons so as to reduce the decay time of the scintillation light to increase the counting rates.

These detectors allow the voxel resolution of about 4-6 $\mathrm{mm}$ due to the improved front end electronics of the scanner but these traditional PET's photomultipliers are much larger than the detectors which are limiting the further voxel resolution. The current research is proceeding to improve the Voxel resolution.

The Time of Flight- PET advanced in the 2000s after a long time internal of 20years as TOF-PET was started in 1980s the current clinical TOF-PET-CT has time of flight in the range 500-600 ps [4]. For this process to occur it needs a fast scintillator. The scintillators used in TOF-PET-CT are LSO, LYSO, and $\mathrm{LaBr}_{3}$, and the new scintillators are $\mathrm{LuI}_{3}$ and $\mathrm{LuAg}$ (CE or Pr) [24]

Properties of Scintillators used in TOP-PET [4]

Table 5
\begin{tabular}{|l|l|l|l|}
\hline Property & LYSO $(10 \% \mathrm{Y})$ & $\mathrm{LSO}$ & $\mathrm{LaBr}_{3}$ \\
\hline Attenuation coefficient $\left(\mathrm{cm}^{-1}\right)$ & 0.86 & 0.90 & 0.47 \\
\hline Decay Time (ns) & 40 & 40 & 27 \\
\hline Light Output (Photons/MeV) & $25-30,000$ & $25-30,000$ & 60,000 \\
\hline
\end{tabular}

\section{2D and 3D PET Imaging}

Generally, Tungsten is used as a collimator which is used to separate several rings of detectors. With collimation 2D data are obtained. The separation of collector rings improves the line of responses (LOR). In the case of $3 \mathrm{D}$ due to a large number of true coincidence in FOV makes it difficult for data analysis and construction of image i.e. the detection of unwanted scattered pattern increases from $10 \%$ to $40 \%$. Current detectors can only be fully 2D or fully 3D. The accidental counts of coincidence from other part reduce the clarity in case of 3D. So 2D is more useful for medical diagnosis which is helpful for Cognitive studies. The PET must be able to distinguish two consecutive events and the time of response for this event is called dead time. Due to modernization in current electronics the pileups in 3D modes has been reduced so as newer models of PET/CT scanners are developed. Recently a cardiac Rb-82 imaging has been done in 3D mode surpassing substantially $2 \mathrm{D}$ image in photon detection. [7,4]

A fig 3 [12] of four ring imaging system with and without separating plane. Where A is strictly 2D, B is modified 2D allowing LOR, C is fully 3D without septa and allowing LOR [12] 


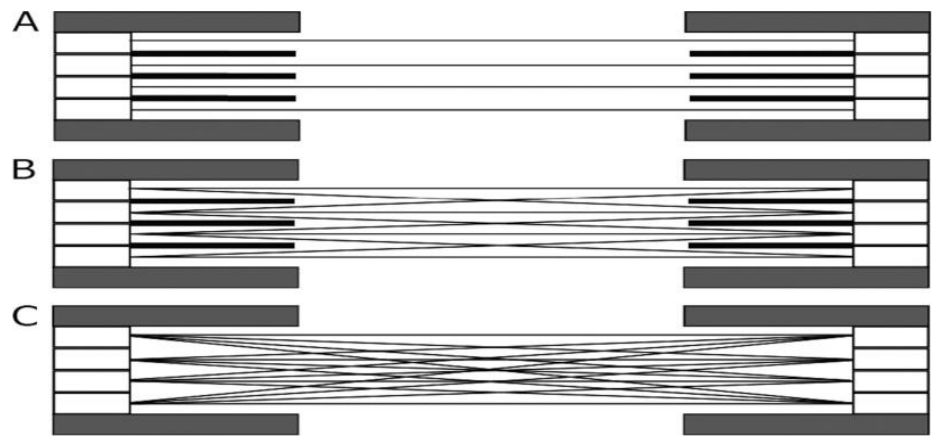

Figure3 [12]

The PET recently underwent a design change making it a digital PET. They are the avalanche photodiodes or smaller silicon digital photo multiplier. Each of which can act as single photon counter. Temporal resolution is really good in this photodiodes making it suitable for Time-of-flight (TOF) acquisition. They are also insensitive to electromagnetic which make it useful in the multimodal integration of PET, MRI, EEG etc, With that the digital PET spatial resolution can go up to $4 \mathrm{~mm}$ voxel. Still the photo detectors are required in PET. Recently the digital cadmium zinc telluride SPECT detectors are used. In future, it may be possible to go fully digital where photo conversion to visible light is not required.[4]

\section{Advancement In MRI}

THE MRI SYSTEM

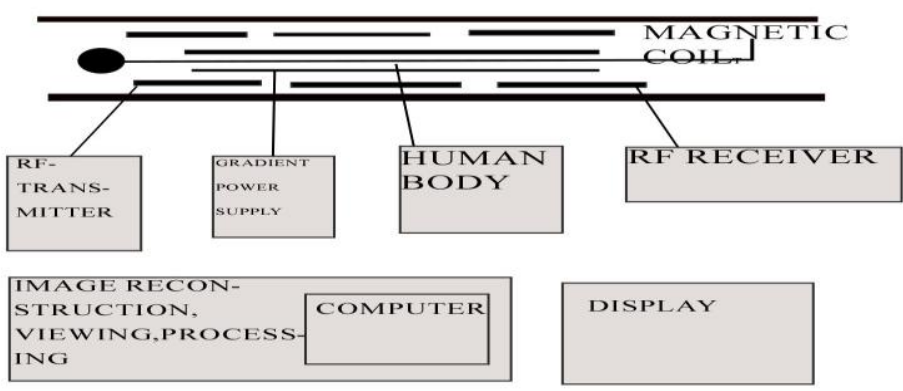

Figure 4

The MR image was first published in the year 1973. There is various level of advancements are based on the needs. As the name suggest the important component in MRI is Magnet. Homogeneous magnetic fields are produced by the ideal MRI's but in practical, it's tough to achieve the homogeneity.

The MRI uses various types of magnets [13] they are Superconducting, resistive and permanent magnets.

Recently $\mathrm{Nb}-\mathrm{Ti}$ is used as a superconducting material. These superconducting magnets can go from $0.5 \mathrm{~T}-2.0 \mathrm{~T}$. Nowadays even 3.0T superconducting magnets are also incorporated in MRI. There are also gradient magnets inside the MRI. These gradient magnets are useful in distorting the homogeneous magnetic field in the patient's body so as to create a gradient field which is controllable. Generally, MRI uses three gradient magnets. These are the magnets which produce noise during the scan. The magnetic field of gradient Magnets can fall within the range of 180 gauss to 270 gauss

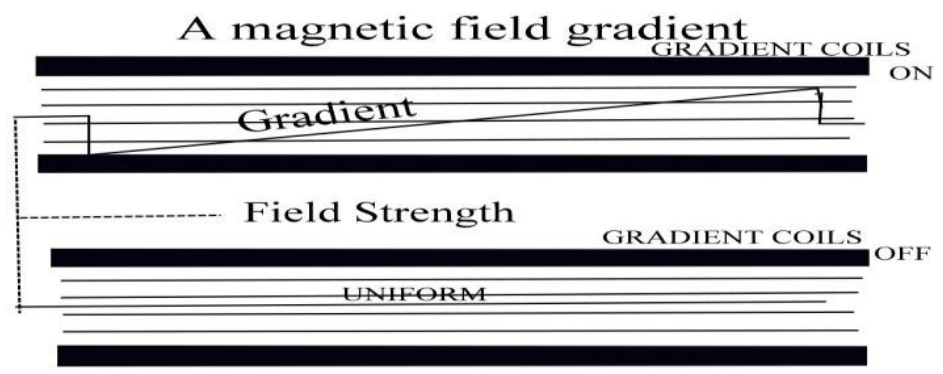

[13] Figure 5 


\subsection{The Radio frequency system.}

The Radio Frequency systems are also a core component in the MRI. The RF coils are incorporated between the magnets. These RF coils have the ability to transmit and receive the signals between the body. There are three basic designs of RF coils. Where one may be used in transmitting and other in receiving but sometimes for both transmission and receiving the same coil is used. The selection of coil solely depends on the experiment that is to be performed. The basic designs of three RF coils are Head, Body, and surface.[13]

The RF polarizer plays another important role in MRI where they can be polarized linearly or circularly. In recent times due to advancement circular polarizer mode is used so as only half of RF energy is required than that of the linear ones. In circularly polarized mode quadrature coils are used where the coils are separated by $90^{\circ}[13]$

Another current advancement in MRI is UTE imaging [13] i.e. Ultra short echo-timing imaging. To achieve shorter Echo time the properties like grater gradient, K-space is to start at center and self-refocused RF pulses to minimize the dead time are achieved. This enabled MRI to image the cortical bones which ones was thought to be not possible.

The functional Brain imaging can be done for both cognitive and affective processes. Which can be fulfilled using the currently available Functional Magnetic Resonance Imaging (fMRI). In fMRI there is a cutting edge state of art equipment called BOLD (blood oxygenation level dependent) fMRI, it measures the ratio of oxygenated to deoxygenated level of hemoglobin in the blood. Thus the Nuclear physics advancement led to BOLD fMRI which doesn't measure the neuronal activity instead it measures metabolic demands of active neurons.

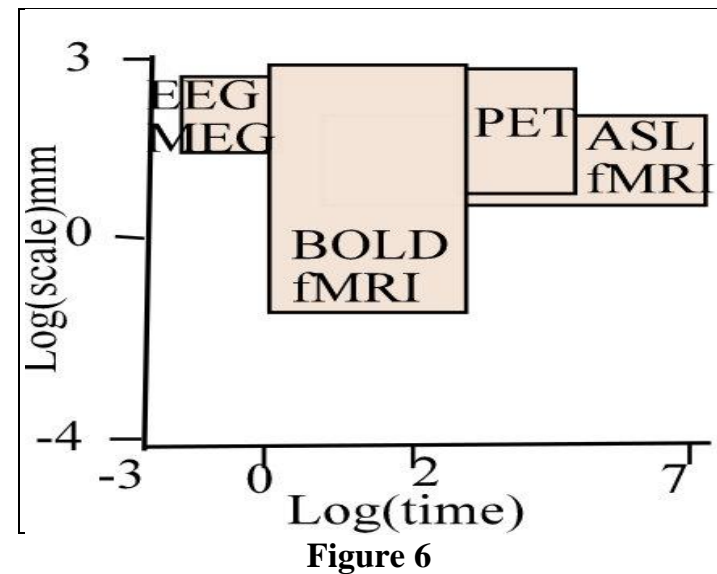

\section{Physics and Measurements Behind The MR Image Formation}

The number of K-space measurements makes up for the spatial resolution while the longitudinal and transverse relaxation time make up for the temporal resolution. Generally, longitudinal relaxation is T1 and Transverse relaxation is T2. The image contrast depends on how often we excite the nuclei (TR) and how fast we collect the data (TE)

The measured signal is

$$
M_{0}\left(1-e^{-T R / T 1}\right) e^{-T E / T 2}
$$

When coming to the spatial resolution in $\mathrm{K}$-space the measurements are in frequency domain made over a finite area. The images are obtained from K-space to image space using Inverse Fourier transforms.

The recent advancements in computational and nuclear physics related to the MRI and system controllers, Statistical tools as well as the software are designed in such way to pre-process and remove artifacts, Visualization, Slice Time correction, Motion correction, Physiological correction, Co-registration, Normalization, Spatial Filtering and Temporal filtering.

\section{Introduction of PET/MR System}

The recent advancement in the field of Imaging Technique is the multimodal integration of various imagers and scanners though one interfere over the other system at the time of scanning, that difficulty has been overthrown by the state of art technologies which made the PET/MR Possible $[17,18,19]$. The PET detectors are arrayed around in the cylinder which are then inserted into the MR Scanner [16]. Here the analog photomultipliers tubes are replaced with high-speed solid state avalanche photo diode detectors [15]. Each 
detector block consists of $3 \times 3$ array 64 crystals of silicon APD's. One of the important physics cited in PET/MR is the reduction of effective positron range and eventually improving the quality of the image. This imager is primarily used in neurology and oncology. The usage of this device in neurology increases the potential of cognitive studies with better results. Reducing the effective positron range currently has less application because ${ }^{18} \mathrm{~F}$-tracers are already having short range so, this may be useful for future advancement in nuclear physics to find a new tracer with longer positron range. e.g. ${ }^{82} \mathrm{Rb}$ in cardiac imaging.[4,20,21,22,23]

\section{Conclusion}

The current advancement in nuclear physics lead to the development of state of art equipment which is useful for mankind like PET, MRI, PET/CT, PET/MR etc., The gist of the advancement in radioactive tracers, magnets, Radio frequency systems, fMRI, BOLD fMRI, and the recently developed PET/MR systems has been discussed. The role of nuclear physics not only includes the construction of equipment and finding new radioactive tracers but also the methods of data acquisition from the equipment, image reconstruction using Fourier transforms, inverse Fourier transforms in K-Space and Image space and also using various statistical tools to reduce the signal to noise ratio. As discussed earlier the Equipment can only rely on the nuclei. So the data are obtained by exploiting the quantum physics principles like spin etic, The Current advancement in Nuclear physics lead to the big leap in the cognitive studies as both the temporal and spatial images. Can be obtained with equipment like MRI and PET. In future as the nuclear physics advances the Voxel by voxel Quality of PET and MRI increases. Which will lead to better imaging of brain that are useful for better cognitive studies

\section{Reference}

[1]. Brownell, G. L., \& Sweet, W. H. (1953). Localization of brain tumors with positron emitters. Nucleonics, 11(11), 4045 .

[2]. Legacy, A. V. (2010). Biological and Environmental Research in the Atomic Age. US Department of Energy, The Office of Biological and Environmental Research, 25-26.

[3]. ROBERTSON J.S., MARR R.B., ROSENBLUM M., RADEKA V., and YAMAMOTO Y.L., “32-Crystal positron transverse section detector, in Tomographic Imaging in Nuclear Medicine, Freedman GS, Editor. 1983, The Society of Nuclear Medicine: New York. pp. 142-153

[4]. Vandenberghe, S., Mikhaylova, E., D’Hoe, E., Mollet, P., \& Karp, J. S. (2016). Recent developments in time-offlight PET. EJNMMI physics, 3(1), 1.

[5]. Surti, S., Kuhn, A., Werner, M. E., Perkins, A. E., Kolthammer, J., \& Karp, J. S. (2007). Performance of Philips Gemini TF PET/CT scanner with special consideration for its time-of-flight imaging capabilities. Journal of Nuclear Medicine, 48(3), 471-480.

[6]. Karp, J. S., Surti, S., Daube-Witherspoon, M. E., \& Muehllehner, G. (2008). Benefit of time-of-flight in PET: experimental and clinical results. Journal of Nuclear Medicine, 49(3), 462-470.

[7]. Schmitz, Ruth, et al. "The physics of PET/Ct scanners." PET and PET/CT: a clinical guide 3 (2005).

[8]. Bailey, D. L., Barthel, H., Beyer, T., Boellaard, R., Gückel, B., Hellwig, D., ... \& Scheffler, K. (2013). Summary report of the first international workshop on PET/MR Imaging, March 19-23, 2012, Tübingen, Germany. Molecular Imaging and Biology, 15(4), 361-371.

[9]. Einstein, A. J., \& Knuuti, J. (2012). Cardiac imaging: does radiation matter?. European heart journal, 33(5), 573578.

[10]. Mankoff, D. A., \& Pryma, D. A. (2014). The contribution of physics to Nuclear Medicine: physicians' perspective on future directions. EJNMMI physics, 1(1), 1.

[11]. MacManus, M., Nestle, U., Rosenzweig, K. E., Carrio, I., Messa, C., Belohlavek, O., ... \& Watanabe, N. (2009). Use of PET and PET/CT for radiation therapy planning: IAEA expert report 2006-2007. Radiotherapy and oncology, 91(1), 85-94.

[12]. Slomka, P. J., Pan, T., \& Germano, G. (2016, January). Recent advances and future progress in PET instrumentation. In Seminars in nuclear medicine (Vol. 46, No. 1, pp. 5-19). WB Saunders.

[13]. Sprawls, P. (2000). Magnetic resonance imaging: principles, methods, and techniques. Medical Physics Publishing.

[14]. Tyler, D. J., Robson, M. D., Henkelman, R. M., Young, I. R., \& Bydder, G. M. (2007). Magnetic resonance imaging with ultrashort TE (UTE) PULSE sequences: technical considerations. Journal of Magnetic Resonance Imaging, 25(2), 279-289.

[15]. Judenhofer, M. S., Wehrl, H. F., Newport, D. F., Catana, C., Siegel, S. B., Becker, M., ... \& Klingel, K. (2008). Simultaneous PET-MRI: a new approach for functional and morphological imaging. Nature medicine, 14(4), 459465.

[16]. Levin, C., Glover, G., Deller, T., McDaniel, D., Peterson, W., \& Maramraju, S. H. (2013). Prototype time-of-flight PET ring integrated with a 3T MRI system for simultaneous whole-body PET/MR imaging. Journal of Nuclear Medicine, 54(supplement 2), 148-148.

[17]. Balyasnikova, S., Löfgren, J., de Nijs, R., Zamogilnaya, Y., Højgaard, L., \& Fischer, B. M. (2012). PET/MR in oncology: an introduction with focus on MR and future perspectives for hybrid imaging. American journal of nuclear medicine and molecular imaging, 2(4), 458. 
[18]. Hirsch, F. W., Sattler, B., Sorge, I., Kurch, L., Viehweger, A., Ritter, L., ... \& Till, H. (2013). PET/MR in children. Initial clinical experience in paediatric oncology using an integrated PET/MR scanner. Pediatric radiology, 43(7), 860-875.

[19]. [Rauscher, I., Eiber, M., Souvatzoglou, M., Schwaiger, M., \& Beer, A. J. (2014). PET/MR in oncology: non-18FFDG tracers for routine applications. Journal of Nuclear Medicine, 55(Supplement 2), 25S-31S.

[20]. Rischpler, C., Nekolla, S. G., Dregely, I., \& Schwaiger, M. (2013). Hybrid PET/MR imaging of the heart: potential, initial experiences, and future prospects. Journal of Nuclear Medicine, 54(3), 402-415.

[21]. Ratib, O., Nkoulou, R., \& Schwaiger, M. (2013). Cardiovascular clinical applications of PET/MRI. Clinical and Translational Imaging, 1(1), 65-71.

[22]. Quick, H. H. (2014). Integrated PET/MR. Journal of Magnetic Resonance Imaging, 39(2), 243-258.

[23]. Adenaw, N., \& Salerno, M. (2013). PET/MRI: Current state of the art and future potential for cardiovascular applications. Journal of Nuclear Cardiology, 20(6), 976-989.

[24]. Lewellen, T. K. (2008). Recent developments in PET detector technology. Physics in medicine and biology, 53(17), R287. 